\title{
The personal recovery movement in Singapore - past, present and future
}

Jonathan Han Loong Kuek ${ }_{B P_{S y}(H o n s)}$, Angelina Grace Liang ${ }^{2}{ }_{B S c}\left(P_{s y c h}\right)$, Ting Wei $\underline{G o h}^{3}{ }_{B A}\left(P_{S y c h}\right)$, Daniel $\underline{\text { Poremski }}{ }^{4}{ }_{P h D}$, Alex $\underline{\mathrm{Su}}{ }^{5}$ MMED (Psych), Hong Choon $\underline{\text { Chua }}{ }^{6}{ }_{M M E D}\left(P_{s y c h)}\right.$

\begin{abstract}
The personal recovery movement is beginning to gain traction within Singapore's mental healthcare systems. We believe it is timely to give a broad overview of how it developed and provide suggestions on how it can evolve further. From the early custodial care in the 1800 s to the community-centric programmes of the 1900 s and early 2000 s, we now find ourselves at the forefront of yet another paradigm shift towards a more consumer-centric model of care. The following decades will allow personal recovery practitioners and researchers to innovate and identify unique but culturally appropriate care frameworks. We also discuss how the movement can continue to complement existing mental healthcare systems and efforts.
\end{abstract}

Ann Acad Med Singap 2021;50:911-4

Keywords: Asia, legislation, lived experience, mental health services, personal recovery

Early psychiatric conceptions of successful mental health recovery traditionally focused on reducing or eradicating symptoms. ${ }^{1}$ The personal recovery movement of the early 1990s in the US prioritised a more holistic and consumer-driven understanding of recovery. ${ }^{2}$ This paradigm shift emphasises that recovery is best defined by people with lived experiences, and their inputs during care provision are greatly valued. ${ }^{2}$ Central to this belief is the active involvement of people experiencing mental health conditions and the spirit of shared decision-making. Hence, practitioners who subscribe to such a worldview view themselves as supporters or guides who journey alongside these individuals, instead of people directing the care process. ${ }^{1}$ Such an approach has resulted in individuals experiencing mental illness being more empowered and better able to manage their conditions. However, this view of recovery predominantly gained traction within Western countries and remains in its nascent stage in most Asian societies. ${ }^{3}$ Across most parts of Asia, mental health conditions are still viewed as biological diseases, and recovery involves eliminating visible symptoms, regaining control of their lives, and reintegrating into society as a contributing individual., ${ }^{4,5}$ In Singapore, although there exists a diverse landscape of mental health research, ${ }^{6-8}$ the personal recovery framework is still a new and growing movement. Therefore, it is timely to discuss its evolution and highlight future directions for its development.

Early mental health services in Singapore (1800s to 1960s). In the $1840 \mathrm{~s}$, the first mental asylums were built to accommodate an increasing number of patients and operated on a custodial model of care. ${ }^{9}$ However, these asylums doubled up as prison compounds for inmates and were often overcrowded. Coupled with high rates of diseases such as tuberculosis, dysentery and cholera, mortality rates among patients were high. ${ }^{10}$ This situation persisted until 1928 when Woodbridge Hospital was constructed to provide dedicated treatment for mentally ill patients. Singapore physicians were also encouraged to study abroad in the UK and Australia to develop their mental healthcare models. However, personal recovery and consumer empowerment were

\footnotetext{
${ }^{1}$ Susan Wakil School of Nursing and Midwifery, Sydney Nursing School, Faculty of Medicine and Health, The University of Sydney, Sydney, Australia

${ }^{2}$ School of Psychological Sciences, Monash University, Melbourne, Australia

${ }^{3}$ Department of Psychology, University at Buffalo, The State University of New York, Singapore Institute of Management Global Education, Singapore

${ }^{4}$ Health Intelligence Unit, Institute of Mental Health, Singapore

${ }^{5}$ Office of Care Transformation, Institute of Mental Health, Singapore

${ }^{6}$ Medical Board, Khoo Teck Puat Hospital, Singapore

Correspondence: Mr Jonathan Han Loong Kuek, Susan Wakil School of Nursing and Midwifery, Sydney Nursing School, Faculty of Medicine and Health, The University of Sydney, Camperdown New South Wales 2006, Australia.

Email: hkue4882@uni.sydney.edu.au
} 
limited during these early years in Singapore. Gradually, a shift towards a more community-oriented mental healthcare occurred in the late 1960s.

Shift to community mental healthcare $(1960 \mathrm{~s}$ to 2009). The Singapore Association for Mental Health (SAMH), formed in 1968, was the nation's first community-based mental healthcare organisation. It took the first step away from institutionalised care to providing more community and social support. In 1986, the Singapore Anglican Community Services (SACS) offered skills and work opportunities for persons with mental health conditions. Involving stakeholders such as educational institutes, families, caregivers, social services and corporate organisations, SAMH and SACS bridged the gap between medical services and community care. ${ }^{11}$ Woodbridge Hospital also provided community psychiatric nurse services from the 1970s that consisted of community-based support and occasional treatment to its discharged patients. In the 2000s, a concerted push by Singapore's Ministry of Health resulted in various nationwide community-based mental health initiatives by the Institute of Mental Health (IMH, previously named Woodbridge Hospital). These initiatives include the Early Psychosis Intervention Programme established in 2001, Aged Psychiatry Community Assessment and Treatment Service in 2006, Community Mental Health Team in 2007 and Job Club in 2009. Supplementing these national initiatives were smaller community-based efforts launched by organisations such as SAMH, SACS and Silver Ribbon (Singapore). ${ }^{12}$ While there was a greater emphasis on rehabilitation and community support, this involvement was still predominantly clinician-driven and lacked active involvement from people experiencing mental illness, which is central to a personal recovery movement.

Peer support and the personal recovery movement (2009 to present). In 2009, the Early Psychosis Intervention Programme was the first initiative to formally recognise the contribution of Peer Support Specialists (PSSs), people living with a mental health condition who used their experiences to support others. Shortly after, in 2011, SAMH and SACS collaborated with the Agency for Integrated Care to train PSSs, and SAMH became the first organisation to hire a fulltime PSS in Singapore, followed by IMH and other community organisations in the following years. ${ }^{13}$ Concurrent with these developments, personal recovery literature was also beginning to surface in Singapore, encouraging practitioners to consider an alternate framework (i.e. strengths-based approaches) while supporting people with mental health conditions. ${ }^{14,15}$ In 2014 , the first personal recovery mental health organisation, PSALT Care, was set up to support people with mental health challenges through peer-led support groups and short-term residential respite for individuals without family support.

In 2017, an effort to unify and standardise various peer support-type programmes across the nation was launched by the National Council of Social Service (NCSS). They partnered with IMH and SAMH to launch a Certified Peer Specialist training programme. With over 5 batches of graduates (10-20 participants per batch), it is now the largest peer support training programme in Singapore. In 2018, Resilience Collective was launched with strong support by NCSS to continue promoting peer support services and the personal recovery movement. Additionally, the Community of Peer Support Specialists was also formed as an informal coalition consisting of PSS alumni from the NCSS to continue supporting the development of the discipline. Finally, Beyond the Label, a nationwide anti-stigma campaign was also launched the same year and adopted an overtly personal recovery stance through its presentation of lived experience narratives and emphasis on the centrality of these experiences.

Complementing these initiatives, IMH has increasingly aligned itself with this personal recovery movement through its aspirations and organisational strategy, which attempts to tackle mental health needs of the population and its direct service users. One such initiative was the introduction of a personal recovery-oriented rehabilitation programme to support the needs of their inpatient clients. Additionally, in 2020, The Tapestry Project SG, a peer-led ground-up movement and platform for people to share their mental health lived experiences was also formally registered as a mental health organisation, continuing its empowerment of people with mental illness through storytelling mediums. In addition, literature examining the personal recovery approach also began to emerge in 2019 to 2020, exploring the lived experiences of people with psychosis ${ }^{16}$ and hoarding, ${ }^{17}$ evaluating peer support services ${ }^{18}$ and testing various recovery-oriented measurement tools. ${ }^{19,20}$ It may be observed that the latter half of the 2010s was an indicator of an impending paradigm shift and the beginning of more personal recovery-oriented perspectives being seeded across the mental healthcare landscape in Singapore. Beyond these developments, there have not been any major/sustained efforts to introduce personal recovery principles into existing mental health services and research agendas.

Challenges and the future of the personal recovery movement. Unfortunately, there are still many challenges ahead to successfully implementing more personal 
recovery-oriented frameworks of care. Firstly, while there are pockets of initiatives appearing within the sector, there lacks a concerted effort by higher-level policymakers to make personal recovery the primary lens through which mental healthcare services should be viewed. Overtly listing the importance of a personal recovery perspective within legislation (i.e. in the Mental Capacity Act or Mental Health [Care and Treatment] Act ${ }^{21}$ or mental healthcare policy could encourage all organisations providing mental healthcare to align themselves with the movement. Such legislation has had a profound impact on health services in other countries as it signified the commitment of regulatory bodies towards advancing the personal recovery narrative within mental healthcare settings (e.g. the US and Australia). ${ }^{22,23}$ With an ever-increasing workload, mental healthcare organisations may also lack the resources and incentive to implement or experiment with new care frameworks, preferring to adhere to the status quo. As part of policy shifts, a common concrete vision/goal in hand with financial, manpower and training resources to reward organisations for implementing recoveryoriented practices could serve as further encouragement in this path.

Secondly, there is a lack of research, direction or guidelines for how to introduce more personal recoveryoriented initiatives or principles within existing mental healthcare systems. Given the novelty of the personal recovery movement in Singapore, there remains a dearth of suitable evidence concerning how its principles can be adopted and adapted to fit the country's cultural context. Singapore is a unique country and needs to chart its own path with regards to the personal recovery movement. Hence, researchers need to make a greater effort to generate a larger and more representative pool of personal recovery literature to allow for a clearer understanding of how it can be best introduced. Examples of substantive research include those evaluating attempts to introduce recovery-oriented concepts in different settings, appropriateness of existing recovery-oriented tools, and recovery-oriented models/frameworks that are culturally appropriate.

Thirdly, Singapore has a unique blend of Western and Eastern ideologies, complicating the potential introduction of personal recovery interventions. For example, in an evaluation article on barriers and facilitators of peer supports services in Singapore, the lack of support and clarity of the PSS role was highlighted as barrier to carrying out their job. ${ }^{18}$ From these findings, it was clear that there exists a strong hierarchical culture in the way organisations operate, and mandating orientation for personal recovery models from the top down is insufficient to ensure that individual service users regularly experience recoveryoriented services. Hence, more targeted interventions such as creating safe spaces for discussions on the introduction of personal recovery principles are needed before interventions can be introduced to address the issues raised. Members of the organisation at every level must embrace new models of care to ensure that the entire hierarchy evolves together.

Lastly, negative perceptions and stigma towards mental health conditions held at a societal level impede the introduction of new approaches for viewing mental health conditions. On a personal level, these negative perceptions could hinder the recovery of those directly impacted. Many people experiencing mental health challenges still face discrimination and stigmatisation. Hence, it is reasonable to assume that most Singaporeans with mental health concerns may not attempt to integrate their mental health issues into their larger personal identity, as there still remains a negative association attached to experiencing them. Such a practice is common in Western personal recovery narratives because it serves as a step towards recovery. Nevertheless, we cannot conclusively anticipate how every individual will respond to the introduction of an alternate perspective towards mental health and recovery. Hence, any large-scale introduction of the personal recovery movement needs to be done cautiously and reflexively. Proponents of the perspective will need to engage deeply in discussions with stakeholders and understand the various barriers and facilitators to shifting mindsets. This additional but deepening engagement may mitigate the possibility of tokenistic implementation of personal recovery-oriented initiatives. These concepts and knowledge can also be shared on a smaller scale with circles that may be more accepting or identify with these perspectives, to generate a ripple effect within the broader mental healthcare sector. Involving people experiencing mental health conditions in these efforts could also send a powerful message on solidarity and the need for a change in narrative about mental health conditions.

Conclusion. Mental healthcare in Singapore has significantly evolved over the years. We are now amid the next major transition to an era where consumercentrism and empowerment are core tenets of services. However, we should not rush the movement and should allow it to develop organically, expand gradually, and eventually become the primary framework of support for people with mental health conditions. 


\section{REFERENCES}

1. Anthony WA. Recovery from mental illness: The guiding vision of the mental health system in the 1990s. Psychosoc Rehabil J 1993;16:11-23.

2. Slade M, Williams J, Bird V, et al. Recovery grows up. J Ment Health 2012;21:99-103.

3. Kuek JHL, Raeburn T, Wand T. Asian perspectives on personal recovery in mental health: a scoping review. J Ment Health 2020;11:1-17.

4. Balaji M, Chatterjee S, Brennan B, et al. Outcomes that matter: A qualitative study with persons with schizophrenia and their primary caregivers in India. Asian J Psychiatr 2012;5:258-65.

5. Subandi MA. Bangkit: The processes of recovery from first episode psychosis in Java. Cult Med Psychiatry 2015;39:597-613.

6. Teo JY, Chong SL, Chiang LW, et al. Cost of inpatient rehabilitation for children with moderate to severe traumatic brain injury. Ann Acad Med Singap 2021;50:26-32.

7. Kirk AHP, Chong SL, Kam KQ, et al. Psychosocial impact of the COVID-19 pandemic on paediatric healthcare workers. Ann Acad Med Singap 2021;50:203-11.

8. Long VJE, Liu JCJ. Behavioural changes during the COVID-19 pandemic: Results of a nationwide survey in Singapore. Ann Acad Med Singap 2021;50:222-31.

9. $\mathrm{Ng} \mathrm{BY,} \mathrm{Chee} \mathrm{KT.} \mathrm{A} \mathrm{brief} \mathrm{history} \mathrm{of} \mathrm{psychiatry} \mathrm{in} \mathrm{Singapore.} \mathrm{Int}$ Rev Psychiatry 2006;18:355-61.

10. Teoh JI. History of institutional psychiatric care in Singapore 1862-1967. In: Wagner NN, Tan ES (Eds). Psychological problems and treatment in Malaysia. Kuala Lumpur: University of Malaya Press; 1971.

11. Wei KC, Lee C, Wong KE. Community psychiatry in Singapore: An integration of community mental health services towards better patient care. Hong Kong J Psychiatry 2005;15:132-7.

12. Gun SY, Leong JJY. Social inclusion: the Singapore story in community mental health development, psychiatric rehabilitation and recovery. Asia Pac J Soc Work 2016;26:167-77.
13. Lee YY, Ang S, Chua HC, et al. Peer Support in Mental Health: A Growing Movement in Singapore. Ann Acad Med Singap 2019;48:95-7.

14. Chan SW, Li Z, Klainin-Yobas P, et al. Effectiveness of a peer-led self-management programme for people with schizophrenia: protocol for a randomized controlled trial. J Adv Nurs 2014;70:1425-35.

15. Xie H. Strengths-based approach for mental health recovery. Iran J Psychiatry Behav Sci 2013;7:5-10.

16. Vaingankar JA, Cetty L, Subramaniam M, et al. Recovery in Psychosis: Perspectives of Clients With First Episode Psychosis. Ann Acad Med Singap 2020;49:186-98.

17. Subramaniam M, Jeyagurunathan A, Devi F, et al. An exploratory study on the lived experiences of hoarding in Singapore. Health Soc Care Community 2020;28:456-66.

18. Kuek JHL, Chua HC, Poremski D. Barriers and facilitators of peer support work in a large psychiatric hospital: a thematic analysis. Gen Psychiatr 2021;34:e100521.

19. Chua YC, Wong HH, Abdin E, et al. The Recovering Quality of Life 10-item (ReQoL-10) scale in a first-episode psychosis population: Validation and implications for patient-reported outcome measures (PROMs). Early Interv Psychiatry 2020;15:1127-35.

20. Lim M, Li Z, Xie H, et al. An Asian study on clinical and psychological factors associated with personal recovery in people with psychosis. BMC Psychiatry 2019;19:1-13.

21. Poremski D, Alexander M, Fang T, et al. Psychiatric Advance Directives and their relevance to improving psychiatric care in Asian countries. Asia Pac Psychiatry 2020;12:e12374.

22. Australian Health Ministers' Advisory Council. A national framework for recovery-oriented mental health services: policy and theory. Commonwealth of Australia, August 2013. Available at: https:// www.health.gov.au/resources/publications/a-national-frameworkfor-recovery-oriented-mental-health-services-policy-and-theory. Accessed on 11 August 2021.

23. Davidson L. The recovery movement: implications for mental healthcare and enabling people to participate fully in life. Health Aff (Millwood) 2016;35:1091-7. 